\title{
A validação brasileira do Questionnaire of Smoking Urges
}

\author{
Brazilian validation of the \\ Questionnaire of Smoking Urges
}

\begin{abstract}
1 Pontifícia Universidade Católica do Rio Grande do Sul, Porto Alegre, Brasil. 2 WP Centro de Psicoterapia Cognitivo-Comportamental, Porto Alegre, Brasil.

Correspondência R. B. Araujo WP Centro de Psicoterapia Cognitivo-Comportamental. Rua Santa Cecília 1556,

Porto Alegre, RS

90420-040, Brasil.

renataudbrasil@hotmail.com
\end{abstract}

\begin{abstract}
This study was designed to validate the Brazilian version of the Questionnaire of Smoking Urges (QSU). The design was experimental, and participants were randomly distributed in groups of zero, 30, and 60 minutes of tobacco abstinence. The total sample was 201, with 67 males and 134 females, age ranging from 18 to $65(M=$ 38.15). The instruments applied, besides QSU, were the Social and Demographic Data Form, Visual Analogue Scale to evaluate craving, Fagerström Test for Nicotine Dependence, and Beck Anxiety and Depression Inventories. Total variation in the two-factor factorial analysis was $57.76 \%$, with a high inter-correlation ( $r=0.726$; $p=0.000$ ). Factor 1 represented the anticipated relief of negative affect, nicotine abstinence symptoms, and urgent and overwhelming desire to smoke. Factor 2 represented the desire to smoke and the anticipation of smoking pleasure. Such results are contrary to those found in the original QSU. The Brazilian version proved to be an adequate and reliable instrument that can be used in both research and patient treatment.
\end{abstract}

Tobacco; Questionnaires; Validation Studies
Renata Brasil Araujo 1,2

Margareth da Silva Oliveira 1

Maria Augusta Mansur ${ }^{2}$

\section{Introdução}

O tabagismo é um comportamento dependente que vem aumentando os índices de morbidade-mortalidade no mundo inteiro ${ }^{1}$. Apesar do conhecimento dos malefícios oriundos do uso do tabaco, a interrupção de seu consumo não é um processo fácil, sendo influenciado pelo craving - "fissura" - por esta substância 2 .

Beck et al. 2 e Marques \& Seibel 3 descrevem o craving como um forte desejo de consumir uma substância, que pode ocorrer durante seu uso, na fase de desintoxicação, ou ainda após uma interrupção mais prolongada, sendo um dos sinais de alerta que poderia levar a um lapso ou recaída. Marlatt \& Gordon 4 diferenciam o termo craving de "compulsão" (Urge), sendo a segunda um comportamento decorrente da vontade subjetiva causada pela primeira. Neste caso, o objetivo do impulso seria o de reduzir o craving e buscar um estado de relaxamento. Kozlowski \& Wilkinson 5 também distinguem esses termos, porém definem urge como um continuum do desejo de consumir uma substância e craving como um estágio específico no qual este desejo é intenso. Tiffany \& Drobes 6 , no entanto, argumentam que são poucas as evidências de que os tabagistas consigam fazer esta distinção entre urge e craving na prática clínica e, em função disto, utilizam estes dois termos como sendo correspondentes. 
Devido a importância do craving no tratamento de comportamentos adictivos, alguns autores preocuparam-se em estudar mais a respeito deste tema e desenvolver instrumentos para a sua mensuração $6,7,8$.

No Brasil, não existem instrumentos de avaliação de craving em tabagistas validados, portanto a validação do Questionnaire of Smoking Urges (QSU) 6 é relevante para um maior aprofundamento deste contructo.

O QSU foi desenvolvido com o objetivo de avaliar o craving em dependentes do tabaco. Além de um somatório total de pontos, ela avalia quatro categorias distintas: desejo de fumar; antecipação de resultado positivo; alívio dos sintomas de privação ou afeto negativo e intenção de fumar, assim como dois fatores, o primeiro, relacionado à intenção principal, desejo de fumar e antecipação do prazer de fumar (Fator 1) e, o segundo, formado pela antecipação do alívio do afeto negativo, dos sintomas da abstinência da nicotina e pelo desejo urgente e arrebatador de fumar (Fator 2) 6 .

Na sua validação, Tiffany \& Drobes ${ }^{6}$ pesquisaram 230 tabagistas da população geral e os dividiram pelos níveis de privação de 0,1 ou 6 horas. Foi realizada uma análise fatorial que verificou que a solução de dois fatores era a mais adequada e, segundo a Análise de Variância, os escores de craving aumentavam em função do tempo de privação. Na validação original não foi realizada análise fatorial das categorias, somente dos dois Fatores. $\mathrm{O} \alpha$-Cronbach dos Fatores 1 e 2 foram respectivamente 0,95 e 0,93 . Willer et al. 9, porém, ao utlizarem o QSU comparando privados ou não do tabaco, avaliaram que os não privados pontuavam mais no Fator 1, enquanto que os privados, no Fator 2. Eles relacionaram o Fator 1 às propriedades de reforço positivo do cigarro e, o Fator 2 às propriedades de reforço negativo. Tiffany \& Drobes 6 não fizeram esta relação, porém destacaram as características reforçadoras positivas do Fator 1 e fizeram uma observação de que o Fator 2 é predominantemente reforçador negativo, sendo, as questões referentes ao desejo, intenção e expectativas de resultado positivo, distintas das encontradas no Fator 1 à medida em que dizem respeito à urgência em fumar.

A validação francesa do QSU 10 dividiu os sujeitos em grupos de 60 e 90 minutos de privação do tabaco e seus dois fatores tiveram resultados semelhantes ao questionário original. Davies et al. 11, na validação inglesa, também encontraram escores parecidos com os da versão original nos dois fatores. Um breve período em abstinência, nesse estudo, elevou o Fator 1 e não o Fator 2. Já os autores da validação ale- mã 12 , consideraram os dois fatores igualmente sensíveis à manipulação do tempo em abstinência.

Uma pesquisa realizada na Itália 13 e a validação espanhola 14 são os estudos mais recentes com o QSU: enquanto a primeira demonstrou sua sensibilidade avaliando o craving em sujeitos que utilizavam adesivos de nicotina, a segunda comprovou que a presença de itens negativos não foi a responsável pela configuração do QSU em dois fatores.

A versão original do QSU demonstrou ser capaz de capturar a multidimencionalidade das medidas do craving, sendo fácil seu entendimento e rápida sua aplicação, o que é comprovado por sua ampla utilização em pesquisas em várias partes de mundo 6 .

Em decorrência da utilidade desse instrumento tanto na área de pesquisa como na clínica, o objetivo deste estudo foi realizar a validação da versão brasileira do QSU 6 .

\section{Método}

\section{Delineamento}

Este estudo teve um delineamento experimental, sendo a variável craving manipulada por meio do controle do tempo de abstinência do tabaco.

\section{Sujeitos}

A amostra foi composta por 201 sujeitos e sua coleta foi por conveniência. Os participantes foram alocados, de maneira aleatória, em três grupos: Grupo 1 (zero minutos de abstinência do tabaco), Grupo 2 (30 minutos de abstinência) e Grupo 3 (1 hora em abstinência do tabaco). Uma parte da coleta foi feita entre os funcionários do Hospital Psiquiátrico São Pedro $(n=57)$ e os demais fizeram parte da população geral $(\mathrm{n}=144)$.

Os participantes eram dos sexos masculino ( $n=67)$ e feminino $(n=134)$, preenchiam os critérios para dependência de tabaco pela CID-10 15, estavam utilizando esta substância há, no mínimo um ano $(\mathrm{M}=21,41$ anos; $\mathrm{SD}=12,4)$, tinham um grau mínimo de escolaridade equivalente a quinta série do Ensino Fundamental $(\mathrm{M}=12,2$ anos de estudo; $\mathrm{SD}=2,74)$ e tinham entre 18 e 65 anos de idade $(\mathrm{M}=38,15$ anos; $\mathrm{SD}=$ 11,93). Fumavam, em média, 17,17 cigarros por dia $(S D=11,0)$, sendo que a média de pontos que obtiveram na Escala Fagerström foi de 4,14 $(\mathrm{SD}=2,58)$ o que é considerado por Achutti 16 como um nível leve de tabagismo. Quanto à mo- 
tivação para interromper o uso do tabaco, 14 sujeitos não pretendiam parar, 133 disseram que o fariam "algum dia", 11 no próximo ano, 17 no próximo mês, 9 na próxima semana e 15 tentariam ao final daquele mesmo dia ( $\mathrm{n}=199$; missing = 2).

Além disso, os sujeitos avaliados não eram dependentes ou faziam "Uso Nocivo", de acordo com a CID-10 15, de outras substâncias psicoativas salvo a cafeína. Não utilizavam nenhum tipo de psicofármaco, não estavam abstinentes há mais que 24 horas do tabaco, nem tentando interromper o seu uso.

\section{Instrumentos}

\section{- Questionnaire of Smoking Urges - versão brasileira}

É uma escala com 32 itens que consistem em 19 afirmativas e 13 negativas relacionadas a diferentes conceitualizações do craving pelo tabaco. É pontuada, de acordo com uma escala de likert 7 pontos $(1=$ discordo completamente; $7=$ concordo completamente), podendo ser avaliada pelo seu total de pontos, pontos por categoria e pontos nos Fatores, sendo, este último, o mais utilizado nos estudos internacionais. Os pontos de corte da versão brasileira para o total de pontos da escala são: de 0 a 63 pontos, fissura mínima ; de 64 a 98, leve; de 99 a 138 moderada e 139 ou mais pontos, fissura intensa.

\section{- Ficha com dados sócio-demográficos}

Utilizada para verificar o perfil sócio-demográfico e referente ao padrão de consumo do tabaco, assim como para identificar características importantes para a avaliação da inclusão e exclusão dos indivíduos na amostra como: ter entre 18 e 65 anos de idade, ter estudado no mínimo até a $5 \underline{a}$ série do Ensino Fundamental, preencher critérios para dependência do tabaco pela CID-10 15, estar utilizando essa substância há, no mínimo um ano, não depender de outras substâncias psicoativas, salvo a cafeína, não utilizar medicações psiquiátricas, nem tampouco estar tentando interromper o uso do tabaco.

\section{- Escala analógico-visual para avaliar o craving}

O indivíduo era solicitado a dar uma nota para o seu craving, sendo 0 (zero) a nota mínima (não apresenta craving) e 10 a máxima (apresenta craving muito forte), marcando este valor em uma escala de 10 centímetros. Vários autores utilizaram Escalas Analógico-Visuais para avaliar o craving em suas pesquisas 12,17 , sendo, uma destas, com o objetivo de fazer uma análise convergente com o QSU para sua validação 12 .

\section{- Fagerström Test for Nicotine Dependence}

Desenvolvida por Fagerström 18 e readaptada por Healtherton et al. 19, Fagerström Test for Nicotine Dependence (FTND) é uma escala que consiste em questões referentes ao padrão típico de fumar e que classifica a dependência da nicotina em leve, moderada ou severa. A versão aplicada, neste estudo, foi a validada no Brasil por Carmo \& Pueyo 20. O total de pontos da FTND, bem como a questão 1 ("Quanto tempo você demora para fumar seu primeiro cigarro depois de se levantar pela manhã?") e a questão 2 ("Para você é difícil abster-se e não fumar naqueles lugares onde está proibido como, por exemplo, um hospital, biblioteca, igreja, ônibus etc?") foram utilizadas na validação convergente.

\section{- Inventário Beck de Ansiedade}

O Inventário Beck de Ansiedade (BAI) 21 é uma escala sintomática, composta por 21 itens, destinada a medir a gravidade dos sintomas de ansiedade, que foi validada para o Brasil por Cunha 22.

\section{- Inventário Beck de Depressão}

O Inventário Beck de Depressão (BDI) 23 é destinado a medir a intensidade da depressão, a partir do escore resultante da soma do total de pontos. Assim como o BAI, também foi validado, no Brasil, por Cunha 22.

\section{Procedimentos}

\section{- Validação Semântica}

Foram realizadas, tendo como fundamentação o estudo de Ciconelli 24 e Pasquali 25, a tradução e validação semântica do QSU, as quais seguiram alguns passos:

a) Tradução do instrumento da Língua Inglesa para a Língua Portuguesa realizada por uma Professora de Inglês, graduada em Letras e conhecedora do objetivo da tradução.

b) Aplicação do instrumento traduzido em dez sujeitos com a finalidade de verificar se as questões eram facilmente compreendidas, levantando possíveis dúvidas.

c) Brainstorming: foram reunidos cinco sujeitos e cada item que compõe os instrumentos foi reproduzido verbalmente por eles, sendo questionado quanto à clareza de cada questão. 
d) Back-translation: a primeira tradução foi revertida para o idioma de origem por um nativo de Língua Inglesa, com fluência na Língua Portuguesa e desconhecedor do objetivo da tradução.

e) Tradução do Instrumento: a partir do backtranslation, o QSU foi novamente traduzido para a Língua Portuguesa por uma psicóloga, brasileira, residente nos Estados Unidos da América, com fluência na Língua Inglesa e conhecedora da finalidade da tradução.

f) Nesta fase da validação foi formado um Comitê de Juízes Especialistas, composto por cinco especialistas em dependência química e dois especialistas em validação de instrumentos psicológicos, os quais compararam as versões do instrumento, verificando se seus itens referiamse ao tema "craving", bem como opinaram a respeito de quais dos itens das duas versões na Língua Portuguesa eram os mais adequados. Os resultados foram levantados, sendo produzida a versão em Português do QSU.

g) Estudo piloto: a versão em Português foi aplicada em vinte sujeitos com as características da amostra pesquisada. O objetivo foi verificar se o instrumento estava adequado tanto gramatical quanto funcionalmente. Com as últimas modificações, foi produzida a versão final do QSU, na qual foram acrescentados os números de 1 a 7 acima dos pontos da escala likert que visualmente estariam relacionados a estes números na escala original. Não houve, após o processo de validação semântica, dificuldade na compreensão da escala, sendo seu tempo médio de aplicação equivalente a $3 \mathrm{mi}$ nutos. Em função das divergências quanto ao significado de urge e craving, utilizou-se, no nome da escala, as suas iniciais na Língua Inglesa (QSU), sendo acrescentado o complemento "versão brasileira". O termo craving não foi traduzido como "fissura" em função deste último ser um termo popular que sofre influências regionais e, por não ser comum o seu uso, segundo os juízes deste estudo, ao ser feita referência ao desejo por fumar. Desta forma, craving, no QSU versão brasileira, foi traduzido como "forte desejo".

\section{Coleta de dados}

Após a conclusão do estudo piloto, foram excluídos os protocolos de pesquisa desta fase inicial, sendo, então, iniciada a coleta de dados.

Os participantes que tinham entre 18 e 65 anos de idade, tinham estudado, no mínimo, até a 5a série do Ensino Fundamental, preenchiam critérios para dependência do tabaco pela CID-10 15, estavam utilizando essa subs- tância há, no mínimo um ano, não dependiam de outras substâncias psicoativas, salvo a cafeína, não utilizavam medicações psiquiátricas e que não estavam tentando interromper o uso do tabaco, nesta etapa seguinte, foram avaliados, individualmente, sendo incluída, no estudo, sua ficha com dados sócio-demográficos.

A amostra foi dividida, por sorteio, em três subgrupos: Grupo 1 com 0 (zero) minuto em abstinência, Grupo 2 com 30 minutos e Grupo 3 com 60 minutos. A escolha destes tempos se fundamentou no trabalho de Guillin et al. 10, que demonstrou a sensibilidade do QSU para avaliar as modificações no craving em breves períodos de abstinência.

Os sujeitos que não preencheram nenhum critério de exclusão foram acompanhados, para que fumassem, longe da sala de entrevista, possibilitando que fosse avaliado o exato momento em que a substância foi utilizada, sendo, logo em seguida, aplicados todos os instrumentos. A ordem de aplicação dos instrumentos que avaliavam o craving foi determinada a partir da verificação do grupo ao qual cada participante pertenceria, podendo ser preenchidos no início (Grupo 1), no meio (Grupo 2) ou ao final da aplicação (Grupo 3).

\section{Análise de dados}

As informações coletadas foram organizadas no Programa Statistical Package for the Social Sciences (SPSS) versão 12.0 (SPSS Inc., Chicago, Estados Unidos). A análise exploratória dos dados constou de testes estatísticos Descritivos e de Freqüências. Na validação do QSU foram utilizados o $\alpha$-Cronbach, a análise fatorial e o coeficiente de correlação linear de Pearson. A análise inferencial empregou os testes: quiquadrado, Teste T de Student para amostras independentes, coeficiente de correlação linear de Pearson, análise de variância (ANOVA), análise de covariância (ANCOVA) e regressão linear. O nível de significância utilizado foi o de $5 \%$.

\section{Aspectos éticos}

Esta pesquisa foi aprovada pelos Comitês de Ética em pesquisa da Pontifícia Universidade Católica do Rio Grande do Sul e do Hospital Psiquiátrico São Pedro antes de ser iniciado o Estudo Piloto. A assinatura do Termo de Consentimento Livre e Esclarecido era pré-condição para que os participantes ingressassem neste estudo. 


\section{Resultados}

\section{Caracterização da amostra} distribuída nos três grupos

A amostra total $(\mathrm{n}=201)$ foi distribuída nos três grupos da seguinte forma: No Grupo 1 (0 minuto em abstinência) ficaram 69 sujeitos, no Grupo 2 (30 minutos) 60 sujeitos e, no Grupo 3 (60 minutos) foram alocados 71 sujeitos.

Foi utilizada a ANOVA para comparar esses três grupos quanto às médias de variáveis importantes para a caracterização da amostra: idade, escolaridade, número de cigarros consumidos por dia, idade de início do tabagismo, tempo de tabagismo, tentativas de interromper o uso do tabaco, número de tratamentos feitos com este objetivo, gravidade da dependência do tabaco e sintomas de ansiedade e depressão. Os resultados estão descritos na Tabela 1 e demonstram que não houve diferença significativa entre os grupos com relação a todas as características analisadas $(\mathrm{p}>0,05)$.

Com relação à variável sexo, no Grupo 1, 24 sujeitos eram homens e 46 mulheres, no Grupo 2, 20 eram homens e 40 mulheres e no Grupo 3 , 23 eram homens e 48 mulheres. O Teste quiquadrado foi utilizado para avaliar se havia associação entre o tempo de abstinência e a variável sexo, o que não foi encontrado $\left(\chi^{2}=0,057\right.$; $\mathrm{p}=0,972$ ), não sendo também encontrada associação entre o tempo de abstinência e a motivação para interromper o uso do tabaco $\left(\chi^{2}=12,30\right.$; $\mathrm{p}=0,266$ ).

\section{Análise fatorial}

Com o objetivo de comprovar a adequação dos dados do QSU para a análise fatorial, foi utilizada, após a reversão dos itens invertidos, o Kaiser-Meyer-Olkin (KMO) e o Teste de Bartlett. Os resultados foram, respectivamente, 0,951 e p < 0,05 e demonstraram que a utilização da ANOVA seria adequada para a validação desta escala. Segue, na Tabela 2, a distribuição das questões nos fatores e categorias da escala a partir da utilização da Rotação Promax.

Foi utilizado o critério de Tiffany \& Drobes 6 para a colocação das questões nos Fatores, o qual considerou como pertencentes ao fator itens com carga fatorial igual ou superior a 0,40 , cuja carga no outro fator fosse menor do que 0,25 , devendo a diferença entre ambas ser de, pelo menos, 0,20.

O Fator 1 teve autovalor de 16,07 e variância de 50,22\%, enquanto que o Fator 2 teve autovalor de 2,41 e variância de $7,54 \%$, sendo o total da variância equivalente a 57,76 \% . A correlação entre os dois fatores foi significativa $(r=$ 0,$726 ; p=0,000$ ).

Foram calculadas as medidas do $\alpha$-Cronbach da escala total, dos dois Fatores e das categorias: o $\alpha$ total foi, neste estudo, de 0,97 (32 itens), o do Fator 1, 0,96 (17 itens) e do Fator 2, 0,92 (13 itens). Os valores de $\alpha$ das categorias foram: 0,92 para desejo de fumar (8 itens), 0,90 para antecipação do resultado positivo (8 itens), 0,83 para alívio dos sintomas de abstinência ou afeto negativo (8 itens) e 0,87 para intenção de fumar (8 itens).

Utilizou-se a regressão linear, com o método stepwise, para identificar quais eram, dentre

Caracterização da amostra nos diferentes tempos de abstinência*

\begin{tabular}{|c|c|c|c|c|c|}
\hline Variáveis & 0 minuto & 30 minutos & 60 minutos & $\mathbf{F}$ & $\mathrm{p}$ \\
\hline Idade & $39,29(11,52)$ & $39,15(11,19)$ & $36,20(12,81)$ & 1,49 & 0,229 \\
\hline Anos de estudo & $12,33(2,70)$ & $12,28(2,56)$ & $12,00(2,74)$ & 0,29 & 0,748 \\
\hline Cigarros/dia & $18,66(11,53)$ & $18,00(12,04)$ & $15,00(9,21)$ & 2,22 & 0,111 \\
\hline Idade do início do tabagismo & $16,46(4,34)$ & $16,61(3,39)$ & $17,56(5,83)$ & 1,12 & 0,329 \\
\hline Tempo de tabagismo & $22,83(12,15)$ & $22,76(11,65)$ & $18,89(13,03)$ & 2,28 & 0,105 \\
\hline Tentativas de interromper o uso do tabaco & $2,15(2,36)$ & $1,91(2,27)$ & $1,92(1,85)$ & 0,26 & 0,771 \\
\hline Tratamentos para o tabagismo & $0,16(0,37)$ & $0,10(0,30)$ & $0,10(0,35)$ & 0,66 & 0,518 \\
\hline Escala Fargeström & $4,16(2,81)$ & $4,45(2,33)$ & $3,86(2,53)$ & 0,86 & 0,426 \\
\hline Depressão (Inventário Beck de Depressão) & $8,44(7,71)$ & $9,27(8,74)$ & $8,84(7,49)$ & 0,17 & 0,841 \\
\hline Ansiedade (Inventário Beck de Ansiedade) & $10,23(10,30)$ & $9,66(9,63)$ & $9,29(8,79)$ & 0,16 & 0,852 \\
\hline
\end{tabular}

* Médias (desvio-padrão) 
Questões do Questionnaire of Smoking Urges agrupadas por categorias e associadas à sua distribuição fatorial.

\begin{tabular}{|c|c|c|}
\hline Questões/Categorias & Fator 1 & Fator 2 \\
\hline \multicolumn{3}{|l|}{ Desejo de fumar } \\
\hline Questão 4* & 0,205 & $0,555^{\star \star \star}$ \\
\hline Questão 6* & 0,072 & $0,725^{\star \star \star}$ \\
\hline Questão 13 & $0,826 * \star$ & 0,108 \\
\hline Questão 17* & 0,089 & $0,779 * * \star$ \\
\hline Questão 18 & $0,783^{\star \star}$ & $-0,152$ \\
\hline Questão 20 & $0,732^{\star \star}$ & 0,203 \\
\hline Questão 23 & $0,731^{\star \star}$ & 0,149 \\
\hline Questão 31 & 0,776 ** & 0,149 \\
\hline
\end{tabular}

Antecipação do efeito positivo

Questão 1

Questão 3

Questão 11*

Questão 15

Questão 19

Questão 21*

Questão 22*

Questão 27*

Alívio dos sintomas de abstinência ou afeto negativo

Questão 2

Questão 7

Questão 8*

Questão 12

Questão 14

Questão 24

Questão 26*

Questão 29

\section{Intenção de fumar}

Questão 5

Questão 9

Questão 10*

Questão 16*

Questão 25

Questão 28*

Questão 30

Questão 32*

* Itens reversos;

** Itens do Fator 1;

*** Itens do Fator 2 as quatro categorias do QSU, as variáveis preditoras dos Fatores 1 e 2. O Modelo que melhor explicou o Fator 1 foi o composto por: desejo de fumar, alívio dos sintomas de abstinência ou afeto negativo e intenção de fumar $\left[\mathrm{R}^{2}=0,928\right.$; $\mathrm{F}(3,196)=847,50 ; \mathrm{p}<0,01]$ e o que melhor explicou o Fator 2 foi o formado por: antecipação do efeito positivo, desejo de fumar e intenção de fumar $\left[R^{2}=0,851 ; F(3,196)=373,53 ; p<0,01\right]$. Os valores dos coeficientes beta de cada variável destes dois modelos distribuídos nos fatores pode ser analisado na Tabela 3 . De acordo com o coeficiente de correlação linear de Pearson as quatro categorias do QSU apresentaram-se, quando relacionadas entre si, com correlação positiva e de alta intensidade $(r>0,75$; $\mathrm{p}<0,01)$.

\section{Variações no craving associadas ao tempo de abstinência}

Foi utilizada a ANOVA para comparar as médias do craving nos diferentes períodos de abstinência. As médias comparadas foram as obtidas a partir do escore total do QSU, da pontuação de seus fatores e categorias, e da escala analógico-visual, sendo estes resultados apresentados na Tabela 4.

\section{Análise correlacional}

Foi encontrada, por meio do coeficiente de correlação linear de Pearson, correlação positiva entre o total de pontos do QSU e: escala analógico-visual de avaliação do craving ( $\mathrm{r}=0,692$; $\mathrm{p}=0,000)$, questão $1(\mathrm{r}=0,146 ; 0,039)$ e questão 2 da Fagerström $(r=0,257 ; p=0,000)$, total de pontos da Fagerström $(r=0,206 ; p=0,003)$, sintomas de ansiedade $(r=0,183 ; p=0,011)$, sintomas de depressão $(r=0,181 ; p=0,012)$ e quantidade de cigarros consumida por dia $(r=0,190$; $\mathrm{p}=0,007)$. Porém, não houve correlação entre o total de pontos do QSU e: idade $(\mathrm{r}=-0,102 ; \mathrm{p}=$ $0,151)$, escolaridade $(r=-0,001 ; p=0,986)$, idade de início do tabagismo $(r=0,050 ; p=0,483)$, tentativas de parar de fumar $(r=-0,027 ; p=0,705)$, número de tratamentos para o tabagismo $(\mathrm{r}=$ 0,$055 ; \mathrm{p}=0,438$ ) e tempo de consumo de tabaco $(r=-0,112 ; p=0,117)$. Segue, na Tabela 5 , a comparação das correlações encontradas nos Fatores 1 e 2 do QSU a partir dos cálculos com o coeficiente de correlação linear de Pearson.

Utilizou-se a ANOVA para comparar o $\mathrm{cra}$ ving nos diferentes níveis de motivação para interromper o uso do tabaco. Não houve diferença entre os níveis motivacionais quanto ao total de pontos do QSU $[\mathrm{F}(5,192)=1,901 ; \mathrm{p}=$ 0,096], aos pontos do Fator $1[F(5,193)=1,502$; 
$\mathrm{p}=0,191]$ e aos pontos do Fator $2[\mathrm{~F}(5,192)=$ 2,12; $\mathrm{p}=0,065$ ].

Foi encontrada, segundo o Teste T de Student para amostras independentes, diferença significativa entre os sexos, tanto nos escores totais do QSU ( $\mathrm{t}=-3,01 ; \mathrm{p}=0,003)$ como nos escores do Fator $1(t=-2,81 ; p=0,006)$, do Fator $2(t=-3,24$; $\mathrm{p}=0,001)$, da Escala Analógico-Visual $(\mathrm{t}=-2,16$; $\mathrm{p}=0,032)$ e do BDI $(\mathrm{t}=-2,07 ; \mathrm{p}=0,04)$. As mulheres tiveram médias maiores em todas estas variáveis. Não identificou-se diferença entre homens e mulheres nas demais variáveis pesquisadas: idade, escolaridade, idade de início do tabagismo, tempo de consumo de tabaco, quantidade de cigarros consumida por dia, quantidade de tentativas de parar de fumar, número de tratamentos para o tabagismo, total de pontos da Fagerström e sintomas de ansiedade $(p>0,05)$.

Foi utilizado o teste qui-quadrado para verificar a associação entre sexo e a variável categórica motivação para interromper o uso do tabaco, o que não pôde ser encontrado $\left(\chi^{2}=8,91\right.$; $\mathrm{p}=0,113$ ).

Por intermédio da ANCOVA foi avaliado se seria a depressão a covariante na diferença do craving entre os sexos, o que foi observado quanto ao total de pontos do QSU $[F(1,190)=$ $4,630 ; \mathrm{p}=0,030]$ e ao Fator $1[F(1,190)=10,26$; $\mathrm{p}=0,002]$, não o sendo quanto ao Fator $2[F$ $(1,190)=0,36 ; p=0,549]$ e Escala Analógico-Visual $[F(1,190)=3,55 ; \mathrm{p}=0,061]$.

\section{Discussão}

O QSU demonstrou, na versão brasileira, após o processo de validação semântica, ser um ins- trumento adequado, de fácil e rápida aplicação e com bom nível de confiabilidade. Os valores do $\alpha$-Cronbach tanto da escala total quanto de seus fatores e categorias foram superior a 0,80 , o que é considerado por Rowland et al. 26 , como uma consistência interna plenamente satisfatória, capaz de predizer que os itens avaliam consistentemente o mesmo constructo $(\alpha<$ 0,70 ). Este resultado também foi encontrado na validação de Tiffany \& Drobes 6, na de Cepeda-Benito et al. 14 e de Mueller et al. 12, o que comprova a confiabilidade do QSU em diferentes culturas.

A escala manteve as condições avaliadas em outros estudos 6,10,11,12,13 para capturar as diferenças no craving em breves períodos de

Tabela 3

Variáveis preditoras dos Fatores 1 e 2 do Questionnaire of Smoking Urges.

\begin{tabular}{lcc}
\hline Modelos/Variáveis & Coeficiente (B) & p \\
\hline $\begin{array}{l}\text { Fator 1 } \\
\text { Constante }\end{array}$ & 0,000 \\
Desejo de fumar & 0,436 & 0,000 \\
Alívio dos sintomas de abstinência & 0,404 & 0,000 \\
ou efeitos negativos & & \\
Intenção de fumar & 0,190 & 0,000 \\
& & \\
Fator 2 & & 0,000 \\
Constante & & 0,000 \\
Antecipação do efeito positivo & 0,687 & 0,020 \\
Desejo de fumar & 0,158 & 0,049 \\
Intenção de fumar & 0,107 & \\
\hline
\end{tabular}

Análise de variância (ANOVA) do craving nos diferentes tempos de abstinência*.

\begin{tabular}{|c|c|c|c|c|c|}
\hline Craving & 0 minuto & 30 minutos & 60 minutos & $f$ & $\mathrm{p}$ \\
\hline QSU Total & $90,33(36,89)$ & $107,12(46,28)$ & $119,56(52,53)$ & 7,22 & 0,001 \\
\hline QSU Fator 1 & $38,29(20,33)$ & $46,35(26,38)$ & $54,32(29,59)$ & 6,86 & 0,001 \\
\hline QSU Fator 2 & $47,67(19,77)$ & $55,05(22,77)$ & $58,00(22,77)$ & 4,18 & 0,017 \\
\hline QSU - desejo de fumar & $20,54(11,69)$ & $25,17(14,11)$ & $29,47(16,12)$ & 7,03 & 0,001 \\
\hline QSU - antecipação do efeito positivo & $24,37(10,28)$ & $28,00(13,31)$ & $32,27(15,33)$ & 6,35 & 0,002 \\
\hline $\begin{array}{l}\text { QSU - alívio dos sintomas de abstinência } \\
\text { ou afeto negativo }\end{array}$ & $17,77(8,92)$ & $21,87(11,63)$ & $22,47(11,70)$ & 3,87 & 0,022 \\
\hline QSU - intenção de fumar & $27,64(10,04)$ & $32,08(10,89)$ & $35,34(12,52)$ & 8,31 & 0,000 \\
\hline Escala analógico-visual & $2,81(3,08)$ & $4,03(2,97)$ & $4,82(3,15)$ & 7,59 & 0,001 \\
\hline
\end{tabular}

* Médias (desvios-padrão).

Segundo o teste Turkey, foi encontrada diferença estatisticamente significativa

em todas as variáveis, somente entre os tempos 0 e 60 minutos $(p<0,05)$.

$\mathrm{QSU}=$ Questionnaire of Smoking Urges. 
Tabela 5

Correlações dos Fatores 1 e 2 do Questionnaire of Smoking Urges (OSU) com outras variáveis.

\begin{tabular}{|c|c|c|}
\hline \multirow[t]{2}{*}{ Variáveis } & \multicolumn{2}{|c|}{ Correlações r } \\
\hline & Fator 1 & Fator 2 \\
\hline Escala analógico-visual & $0,643^{*}$ & $0,636^{*}$ \\
\hline Cigarros/dia & $0,197^{\star}$ & $0,182^{*}$ \\
\hline Idade de início do tabagismo & 0,026 & 0,051 \\
\hline Tempo de consumo do tabaco & $-0,085$ & $-0,117$ \\
\hline Tentativas de parar de fumar & $-0,016$ & $-0,064$ \\
\hline Tratamentos para o tabagismo & 0,103 & 0,034 \\
\hline Total de pontos da Fagerström & $0,244^{\star}$ & $0,163^{*}$ \\
\hline Questão 1 da escala Fagerström & $0,188^{\star}$ & 0,104 \\
\hline Questão 2 da escala Fagerström & $0,242^{\star}$ & $0,268^{*}$ \\
\hline Sintomas de ansiedade (Inventário Beck de Ansiedade) & $0,249 *$ & 0,090 \\
\hline Sintomas de depressão (Inventário Beck de Depressão) & $0,249^{\star}$ & 0,076 \\
\hline Total de pontos do QSU & $0,948^{*}$ & $0,898^{*}$ \\
\hline Desejo de fumar & $0,926^{*}$ & $0,859 *$ \\
\hline Antecipação do efeito positivo & $0,849^{*}$ & $0,915^{*}$ \\
\hline Alívio dos sintomas de abstinência ou afeto negativo & $0,898^{*}$ & $0,741^{*}$ \\
\hline Intenção de fumar & $0,856^{\star}$ & $0,805^{\star}$ \\
\hline
\end{tabular}

* $p<0,05$ de abstinência são quesitos avaliados pelo Fator 1 da escala brasileira e estes sintomas aumentam em proporção direta com a quantidade de cigarros utilizada 16 e com o intervalo do consumo do último cigarro 6 , imaginava-se, pelas médias inferiores, nestas variáveis, que o Fator 2, e não o 1, seria o principal.

A amostra brasileira estudada, deve-se enfatizar, tinha um bom nível de escolaridade e, em sua maioria, estava trabalhando, o que faz com que se descarte possíveis carências socioeconômicas muito comuns de serem vivenciadas por grande parte da população brasileira, como a causa deste contraste com os resultados dos estudos internacionais 6,9,10,12,14. Pode-se inferir, então, que esta diferença ocorreu em função de aspectos associados ao afeto negativo - outro item avaliado por este Fator. Os sintomas de ansiedade e depressão, mesmo tendo médias baixas 22 , bem como outros fatores de ordem emocional não pesquisados podem ter interferido nesta distribuição fatorial. Por outro lado, o aumento do número de propagandas antitabaco veiculadas pelo Ministério da Saúde, no Brasil, nos últimos anos, foi bastante significativo, sendo possível que este seja um dos fatores responsáveis por estas diferenças, já que a antecipação do efeito positivo do cigarro é influenciada por expectativas de cunho negativo ligadas às restrições quanto ao uso desta substância 4 .

Além disto, deve-se explicitar que em várias validações do QSU 6,10,14 os tabagistas pesquisados eram mais jovens, fumavam há menos tempo e eram predominantemente do sexo masculino, o que pode influenciar nos sintomas de abstinência e, por sua vez, no craving avaliado pelo Fator 1 de nossa pesquisa.

Na versão brasileira, os dois fatores do QSU, juntos, foram compostos por 30 questões - mais do que as 26 da escala americana 6 - havendo mais questões no Fator 1 do que no Fator 2. Não foi realizada a análise fatorial das quatro categorias do QSU por esta categorização ter sido determinada pelos autores do instrumento original 6 , sem que fosse realizada, também naquele estudo 6 , a análise fatorial correspondente.

Utilizou-se a regressão linear, comparando os resultados com a definição que Tiffany \& Drobes 6 atribuíram a cada um dos fatores na versão original e pode-se perceber que o nosso Fator 1 avalia o desejo de fumar, o alívio dos sintomas da abstinência ou afeto negativo e, com menos intensidade, a intenção de fumar. Verificando as questões da categoria desejo de fumar que compõem este Fator, foi possível identificar o seu caráter de urgência, o que havia sido discriminado por Tiffany \& Drobes 6 . 
Ao ser aplicada a regressão linear ao Fator 2 e às categorias do QSU, encontramos na composição deste fator a antecipação do efeito positivo, o desejo de fumar e a intenção de fumar, tendo um peso maior para a antecipação do efeito positivo. A categoria Alívio dos sintomas de abstinência ou afeto negativo, por esta análise estatística, não compôs o Fator 2, apesar de algumas questões referentes a esta categoria terem sido alocadas como tal na distribuição fatorial.

A questão 1 da Fagerström, os sintomas de ansiedade e de depressão estiveram correlacionados com o Fator 1 e não com o Fator 2, o que comprova a função do primeiro fator referente ao alívio dos sintomas de abstinência ou afeto negativo, sendo este resultado também semelhante, apesar de inverso quanto aos fatores, ao divulgado pelos autores da escala original 6 .

Por meio da análise correlacional pode-se observar que os Fatores 1 e 2 apresentaram, assim como na validação de Tiffany \& Drobes 6 , correlação positiva e de alta intensidade entre si 27, o que demonstra que houve uma influência direta de um sobre o outro, sem que houvesse redundância em suas avaliações, já que os resultados foram abaixo de 0,8028 . Quanto às correlações de cada fator em separado com as categorias, obtiveram-se também, correlações positivas de alta intensidade, porém todas estas foram superiores a 0,80 , exceto a correlação entre a categoria alívio dos sintomas de abstinência ou afeto negativo e o Fator 2, o que mostra o quanto é difícil separar o craving a partir deste tipo de definição dado o seu caráter correlacional.

Não foi identificado, da mesma forma que Willer et al. ${ }^{9}$, uma divisão clara pela qual um fator avalia o reforço positivo e, outro, o reforço negativo. Os resultados da validação brasileira se assemelham mais aos de Tiffany \& Drobes 6 que, apesar de ressaltarem um predomínio do reforço positivo em um fator e do negativo em outro, não consideram estes como excludentes.

A validação covergente do QSU demonstrou que o total de pontos desta escala teve correlações positivas: de alta intensidade com a escala analógico-visual, de muito baixa intensidade com a questão 1 da Fagerström e de baixa intensidade com a questão 2, porém deve-se lembrar que estas três escalas com as quais o QSU foi comparado, não conseguem avaliar o $\mathrm{cra}$ ving em seu caráter multidimensional, fato este defendido por alguns autores 6,17,29.

O craving mensurado pelo total de pontos do QSU apresentou correlações positivas baixas ou muito baixas com a gravidade da depen- dência do tabaco, a quantidade de cigarros consumida por dia, sintomas de ansiedade e depressão, e não teve correlação com o tempo de tabagismo e a motivação para interromper o uso do tabaco, demonstrando o quanto este fenômeno é complexo, já que estas variáveis têm sido freqüentemente associadas ao $\mathrm{cra}$ ving 30,31,32.

Deve-se destacar que o craving não estava correlacionado com idade, o que contrasta com as conclusões de Daughton et al. 33; do mesmo modo, não estava correlacionado com escolaridade, o que é um dado importante à medida em que, nesta amostra, a média de anos estudados foi alta. Apesar deste achado, deve-se ter cuidado quando se avalia esta questão, já que Broms et al. 34 encontraram relação entre escolaridade e maior facilidade para a cessação do uso do tabaco.

Outro ponto interessante foi o de que as mulheres apresentaram craving mais intenso do que o dos homens, porém este resultado foi influenciado por sintomas de depressão no sexo feminino, os quais levaram a um aumento do Fator 1, intensamente relacionado ao alívio dos sintomas de abstinência ou afeto negativo e, concomitantemente, do total de pontos do QSU. É relevante a constatação de que o Fator 2 que avalia predominantemente o desejo de fumar ligado ao prazer atribuído à nicotina, não ter sido influenciado pelos sintomas depressivos ou ansiosos, pois este resultado aponta o sucesso, já destacado em outros estudos 6,9,11, 12,14, da composição de dois fatores do QSU para a avaliação do craving em tabagistas.

\section{Conclusão}

A validação brasileira do QSU pode comprovar as propriedades psicométricas satisfatórias deste instrumento, bem como sua capacidade para avaliar o craving em tabagistas a partir do entendimento deste constructo como multidimensional, o que reflete as vantagens que tem com relação a escalas que avaliam uma única dimensão do craving como a escala analógicovisual.

Desejo de fumar, antecipação do efeito positivo, alívio dos sintomas da abstinência ou do afeto negativo e intenção de fumar, apesar de serem distintas categorias estão inter-relacionadas na configuração do craving, devendo cada uma delas ser analisada não só isoladamente, mas principalmente em conjunto.

Neste estudo, pode-se constatar que a solução de dois fatores do QSU é adequada para a avaliação do craving por sua capacidade discri- 
minante e que o Fator 1, que reflete a intenção principal, está relacionado à antecipação do alívio do afeto negativo e dos sintomas da abstinência da nicotina, bem como ao desejo urgente e arrebatador de fumar, enquanto o Fator 2 se relaciona ao desejo de fumar e à antecipação do prazer de fumar. Mesmo a inversão entre os fatores ocorrida na versão brasileira não chegou a prejudicar sua validade, já que a distribuição das questões manteve-se a mesma da escala original.

Esta validação, deve-se alertar, teve uma amostra com elevado grau de escolaridade, o que pode ter interferido nos resultados, bem

\section{Resumo}

Este estudo teve por objetivo validar a versão brasileira do Questionnaire of Smoking Urges (QSU). Teve delineamento experimental, sendo que seus participantes foram distribuídos, aleatoriamente, em grupos de zero, 30 e 60 minutos de abstinência do tabaco. A amostra teve 201 sujeitos dos sexos masculino $(n=67)$ e feminino $(n=134)$, que tinham entre 18 e 65 anos de idade $(M=38,15)$. Os instrumentos utilizados, além do QSU, foram ficha com dados sócio-demográficos, escala analógico-visual para avaliar o craving, Fagerström Test for Nicotine Dependence $e$ os inventários Beck de ansiedade e de depressão. Observou-se que o total da variância da análise fatorial com dois fatores foi de $57,76 \%$, havendo alta correlação entre ambos $(r=0,726 ; p=0,000)$. O Fator 1 representou antecipação do alívio do afeto negativo, dos sintomas da abstinência da nicotina e desejo urgente e arrebatador de fumar, e o Fator 2 refletiu o desejo de fumar e antecipação do prazer de fumar, resultados inversos aos da versão original. A versão brasileira do QSU demonstrou ser um instrumento adequado e confiável, podendo ser utilizado tanto na pesquisa quanto na clínica. como teve um grau leve de dependência da nicotina, sendo estas, limitações para as quais sugere-se a realização de novas pesquisas com sujeitos com diferentes características sóciodemográficas e com maior padrão de uso do tabaco.

Espera-se, porém, que a versão brasileira do QSU possa ser um instrumento útil não só para os pesquisadores como para os profissionais que atendem dependentes químicos, auxiliando-os na avaliação do craving, com o objetivo de tornar, assim, um pouco menos complicada a difícil manutenção da abstinência.

\section{Colaboradores}

R. B. Araujo participou de todas as etapas da produção do artigo. M. S. Oliveira revisou o artigo. M. A. Mansur participou da coleta de dados.

\section{Agradecimentos}

Este trabalho fez parte da Tese de Doutorado em Psicologia de Renata Brasil Araujo com o apoio financeiro da Coordenação de Aperfeiçoamento de Pessoal de Nível Superior. Ao Dr. David Drobes (Purdue University) e Dr. Stephen Tiffany (Purdue University) pelas autorizações dadas para a validação do QSU no Brasil.

Tabaco; Questionários; Estudos de Validação 


\section{Referências}

1. Gigliotti A, Bonetto DV, Alves GJ. Tabagismo. J Bras Med 1999; 77:2.

2. Beck AT, Wright FD, Newman CF, Liese BS. Cognitive therapy of substance abuse. New York: Gulford Press; 1993.

3. Marques AC, Seibel SD. O craving. In: Seibel SD, Toscano JR, organizadores. Dependência de drogas. São Paulo: Atheneu; 2001. p. 239-48.

4. Marlatt A, Gordon J. Prevenção de recaída - estratégias de manutenção no tratamento de comportamentos adictivos. Porto Alegre: Artes Médicas; 1993.

5. Kozlowski LT, Wilkinson DA. Use and misuse of the concept of craving by alcohol, tobacco, and drug researchers. Br J Addict 1987; 82:31-6.

6. Tiffany ST, Drobes DJ. The development and initial validation of a questionnaire on smoking urges. Br J Addict 1991; 86:1467-76.

7. Sayette MA, Shiffman S, Tiffany S, Niaura R, Martin CS, Shadel W. The measurement of drug craving - methodological aproaches to craving research. Addiction 2000; 95 Suppl 2:S189-210.

8. Sweeney C, Pillitteri J, Kozlowski LT. Measuring drug urges by questionnaire: do not balance scales. Addict Behav 1996; 21:199-204.

9. Willer P, Hardman S, Eaton G. Subjective and behavioural evaluation of cigarette cravings. Psychopharmacology (Berl) 1995; 118:171-7.

10. Guillin O, Krebs MO, Bourdel MC, Olie JP, Loo H, Poirer MF. Validation of the French translation and factorial structure of tiffany and drobes smoking urge questionnaire. Encephale 2000; 26:27-31.

11. Davies GM, Willner P, Morgan MJ. Smoking-related cues elicit craving in tobacco "chippers": a replication and validation of the two-factor structure of the questionnaire of smoking urges. Psychopharmacology (Berl) 2000; 152:334-42.

12. Mueller V, Mucha RF, Ackermann K, Pauli P. Die erfassung des cravings bei rauchern mit einer deutschen version des "questionnaire on smoking urges” (QSU-G). Z Klin Psychol Psychother 2001; 30:164-71.

13. Teneggi V, Tiffany ST, Squassante L, Milleri S, Ziviani L, Bye A. Smokers deprived of cigarettes for $72 \mathrm{~h}$ : effect of nicotine patches on craving and withdrawal. Psychopharmacology (Berl) 2002; 164:177-87.

14. Cepeda BA, Henry K, Gleaves DH, Fernandez MC. Cross-cultural investigation of the questionnaire of smoking urges in american and spanish smokers. Assessment 2004; 11:152-9.

15. Organização Mundial da Saúde. Classificação de transtornos mentais e de comportamento da CID10: descrições clínicas e diretrizes diagnósticas. Porto Alegre: Artes Médicas; 1993.

16. Achutti A. Guia nacional de prevenção e tratamento do tabagismo. São Paulo: Vitrô Comunicação \& Editora; 2001.

17. Singleton EG, Anderson LM, Heishman SJ. Reliability and validity of the Tobacco Craving Questionnaire and validation of craving-induction procedure using multiple measures of craving and mood. Addiction 2003; 98:1537-46.

18. Fagerström KO. Measuring degree of physical dependence to tobacco smoking with reference to individualisation of treatment. Addict Behav 1978; 3:235-41.

19. Heatherton TF, Kozlowski LT, Frecker RC, Fagerstrom KO. The Fargerström Test for nicotine dependence: a revision of the Fagerström tolerance questionnaire. Br J Addict 1991; 86:1119-27.

20. Carmo JT, Pueyo AA. A adaptação do português do Fagerström test for nicotine dependence (FTND) para avaliar a dependência e tolerância à nicotina em fumantes brasileiros. Rev Bras Med 2002; 59:73-80.

21. Beck AT, Steer RA. Beck Anxiety Inventory - manual. San Antonio: Psychological Corporation; 1993.

22. Cunha JA. Manual da versão em português das Escalas Beck. São Paulo: Casa do Psicólogo; 2001.

23. Beck AT, Steer RA. Beck Depression Inventory manual. San Antonio: Psychological Corporation; 1993.

24. Ciconelli RM. Tradução para o português e validação do questionário genérico de avaliação de qualidade de vida "medical outcomes study 36 item short-form health survey (SF-36)" [Tese de Doutorado]. São Paulo: Escola Paulista de Medicina, Universidade Federal de São Paulo; 1997.

25. Pasquali L. Princípios de elaboração de escalas psicológicas. Rev Psiq Clín 1998, 25:206-13.

26. Rowland D, Arkkelin D, Crisler L. Computer-based data analysis: using SPSS in the social and behavioral sciences. Chicago: Nelson-Hall; 1991.

27. Bisquerra R, Sarriera JC, Martinez F. Introdução à estatística - enfoque informático com o pacote estatístico SPSS. Porto Alegre: Artmed Editora; 2004.

28. Ferrans CE, Powers MJ. Psyquiatric assesment of the quality of life index. Res Nurs Health 1992; 15:29-38.

29. Cox LS, Tiffany ST, Christen AG. Evaluation of the brief questionnaire of smoking urges (QSU-brief) in laboratory and clinical settings. Nicotine Tob Res 2001; 3:7-16.

30. Cavallo DA, Pinto A. Effects of mood induction on eating behavior and cigarette craving in dietary restrainers. Eat Behav 2001; 2:113-27.

31. Delfino RJ, Jamner LD, Whalen CK. Temporal analysis of the relationship of smoking behavior and urges to mood states in men versus women. Nicotine Tob Res 2001; 3:235-48

32. Pomerleau CS, Brouwer RJ, Pomerleau OF. Emergence of depression during early abstinence in depressed and non-depressed women smokers. J Addict Dis 2001; 20:73-80.

33. Daughton DM, Fortmann SP, Glover ED, Hatsukami DK, Heatley AS, Lichtenstein E, et al. The smoking cessation efficacy of varying doses of nicotine patch delivery systems 4 to 5 years postquit day. Prev Med 1999; 28:113-8.

34. Broms U, Silventoinen K, Lahelma E, Koskenvuo M, Kaprio J. Smoking cessation by socioeconomic status and marital status: the contribution of smoking behavior and family background. Nicotine Tob Res 2004; 6:447-55.

Recebido em 07/Abr/2005

Versão final reapresentada em 06/Jan/2006 Aprovado em 16/Fev/2006 